\title{
Parameter Estimation Algorithms for Volterra Digital Systems
}

\author{
Wei-Der Chang, Ching-Lung Chi, Shun-Peng Shih, and Bo-Hong Ye
}

\begin{abstract}
In this paper, we will propose a novel parameter estimation algorithm which is based on the modified particle swarm optimization (PSO) algorithm for the Volterra digital system. In the modified PSO algorithm, another adjusting factor is added into the velocity updating formula to enhance the algorithm's search capacity. On the basis of a series of input-output data pairs, we wish that the modified PSO approach can successfully solve for the unknown parameters of the Volterra digital system. The whole design steps based on the modified PSO is presented for parameter estimations. Besides, different sets of algorithm initial conditions are examined to confirm the feasibility and robustness. Finally, simulation results sufficiently reveal that the proposed method can correctly solve for the parameters of the Volterra digital system.
\end{abstract}

Index Terms - Parameter estimation, Volterra digital system, Particle swarm optimization (PSO).

\section{INTRODUCTION}

Parameter estimation is an important topic in the system engineering. If the correct system parameters are obtained by some approaches, the sequential tasks including the controller design and/or the system output forecasting can just proceed. In recent years, lots of researches regarding the parameter estimation have been reported [1]-[6] according to different methods and design targets. In [1], for example, they studied the parameter estimation problem of the Hammerstein nonlinear system using the adaptive filtering technique. A LF-RLS identification algorithm was proposed by filtering the input-output data. In [3], the authors focused on the heavy-tailed stochastic signals generated through continuous-time autoregressive model and the goal is to estimate the parameters of the continuous-time model. For identifying ordinary differential equation models, a two-stage parameter estimation method was presented in [6]. A batch-process model was successfully examined using the proposed design method.

Recently, a large number of evolutionary computations have successively been presented. Among them, the particle swarm optimization (PSO) algorithm proposed by Kennedy and Eberhart is rather popular and often employed [7]. The motivation of the algorithm was basically from the related organization behavior such as fish school and bird flock.

Manuscript received December 18, 2016. This work was supported in part by the Ministry of Science and Technology of Taiwan under Grant MOST 105-2221-E-366-007.

The authors are with the Department of Computer and Communication, Shu-Te University, Kaohsiung, Taiwan (e-mail: wdchang@stu.edu.tw, pipn@stu.edu.tw, spshih@stu.edu.tw,s12115260@stu.edu.tw).
Due to some excellent properties, the algorithm has successfully solved for a variety of engineering optimization problems; for instances, the power system state estimation [8], signature verification [9], multi-objective hull assembly line balancing [10], and constrained optimization problems [11]. In our previous work, a modified version of PSO algorithm was initially developed for the optimal design of nonlinear PID control system [12]. This modified version is to add another adjusting factor into the velocity formula of the algorithm to guide all particle movements. Based on the developed algorithm, a tracking control of nonlinear inverted pendulum system has been successfully implemented by the designed PID controller.

This paper will apply the modified PSO algorithm to the parameter estimation especially for the Volterra digital system. All unknown system parameters are estimated by the proposed method with a series of input-output signal pairs. The remainder of this paper can be summarized as follows. In Section II, the difference equation structure for the Volterra digital system is firstly introduced. In Section III, the modified version of PSO algorithm is addressed in detail, and the whole design steps based on the modified PSO is developed for the parameter estimation of Volterra digital system in Section IV. Section V then is to show several simulation results which examine different sets of algorithm initial conditions. Finally, a brief conclusion is given in Section VI.

\section{Volterra Digital System}

In the digital signal process (DSP), there are two different kinds of digital filter structures: the finite impulse response (FIR) and the infinite impulse response (IIR). The output of the former is influenced only by the present and past input signals, not by the past output signals. Conversely, the output of IIR structure is then affected by both input and output signals. Equation (1) describes a general expression for the FIR digital filter

$$
\begin{gathered}
y[n]=\sum_{k=0}^{M} h[k] x[n-k] \\
=h[0] x[n]+h[1] x[n-1]+\cdots+h[M] x[n-M],
\end{gathered}
$$

where $x$ is the input signal, $y$ is the output signal, $M$ denotes the number of past inputs required, $h[k]$ then represents the system parameter. The Volterra digital system considered in this study is basically an extended version of the FIR digital filter, which can be expressed by the following difference equation [13] 


$$
\begin{gathered}
y[n]=h[0]+\sum_{k=1}^{M} h[k] x[n-k+1] \\
+\sum_{k_{1}=0}^{M-1} \sum_{k_{2}=k_{1}}^{M-1} h\left[k_{1}, k_{2}\right] x\left[n-k_{1}\right] x\left[n-k_{2}\right] \\
=h[0]+h[1] x[n]+\cdots+h[M] x[n-M+1] \\
+h[0,0] x^{2}[n]+\cdots+h[0, M-1] x[n] x[n-M+1] \\
+\cdots+h[M-1, M-1] x^{2}[n-M+1],
\end{gathered}
$$

where $h\left[k_{1}, k_{2}\right]$ denotes the second-order system parameter and also reflects the quadratic properties of the digital system. In this paper, all parameters of the Volterra digital system including $h[k]$ and $h\left[k_{1}, k_{2}\right]$ will be estimated by the developed method which is introduced in the next section.

\section{A MODIFIED VERSION OF PSO ALGORITHM}

Before introducing the modified PSO algorithm, let us consider the general PSO. In the PSO algorithm, all determined system parameters need to be collected and further form a parameter vector as

$$
\Theta=\left[\theta_{1}, \theta_{2}, \cdots, \theta_{N}\right]
$$

where $N$ stands for the number of determined parameters for the optimized problem. Equation (3) is also called the particle in the viewpoint of the PSO algorithm. In addition, let the $i$ th particle's expression be

$$
\Theta_{i}=\left[\theta_{i 1}, \theta_{i 2}, \cdots, \theta_{i N}\right] .
$$

Each particle is guided by the following two updating formulas:

$$
v_{i j} \leftarrow w \cdot v_{i j}+c_{1} \cdot r_{1} \cdot\left(p_{i j}-\theta_{i j}\right)+c_{2} \cdot r_{2} \cdot\left(g_{j}-\theta_{i j}\right),
$$

and

$$
\theta_{i j} \leftarrow \theta_{i j}+v_{i j}
$$

where Equation (5) is the velocity updating formula and Equation (6) is the position updating formula, $\theta_{i j}, p_{i j}$, and $g_{j}$ are the $j$ th position components of the $i$ th particle, the $i$ th individual best particle, and the global best particle, respectively, $v_{i j}$ is the $j$ th velocity component of the $i$ th particle, $w$ is the inertia weight, $c_{1}$ and $c_{2}$ are two positive constants, $r_{1}$ and $r_{2}$ are then two random numbers generated from the interval $[0,1]$ uniformly. The general PSO is just to utilize these two updating formulas to achieve the optimization.

On the other hand, in addition to the individual best $p_{i j}$ and the global best $g_{j}$, another adjusting factor, the local best $s_{j}$, is introduced into the velocity formula of (5) in the modified PSO algorithm. The local best $s_{j}$ means the best particle within the subpopulation where the particle $\theta_{i j}$ belongs to. As a result, the original single population needs to be partitioned into several subpopulations simply by the particle order. The velocity updating formula of (5) is changed to be [12]

$$
\begin{aligned}
v_{i j} \leftarrow w \cdot & v_{i j}+c_{1} \cdot r_{1} \cdot\left(p_{i j}-\theta_{i j}\right) \\
& +c_{2} \cdot r_{2} \cdot\left(g_{j}-\theta_{i j}\right)+c_{3} \cdot r_{3} \cdot\left(s_{j}-\theta_{i j}\right),
\end{aligned}
$$

where $s_{j}$ is the $j$ th position component of the local best, $c_{3}$ is a positive constant, and $r_{3}$ is also a random number selected from $[0,1]$ as well as $r_{1}$ and $r_{2}$. Besides, Equation (6), the position updating formula, still remains. To estimate the unknown parameters of Volterra digital system, the modified version of PSO algorithm is employed.

\section{DESIGN STEPS FOR PARAMETER Estimations}

Fig. 1 shows the overall block diagram of the proposed estimation method for the Volterra digital system, where $x[n]$ is an external input signal, $y[n]$ is the output signal of the Volterra digital system with unknown parameters, $y_{m}[n]$ is the output signal of the Volterra digital model with the same structure as the above Volterra digital system, $e[n]=y[n]-y_{m}[n]$ is the error signal between $y[n]$ and $y_{m}[m]$. When minimizing the error signal by the proposed method, the system parameters can be correctly obtained. Furthermore, we need a proper objective function to evaluate each particle, and here it is simply defined by

$$
O F(\Theta)=\sum_{n=0}^{T-1} e^{2}[n]
$$

where $T$ represents the sampling number. A better particle means that its objective function is smaller.

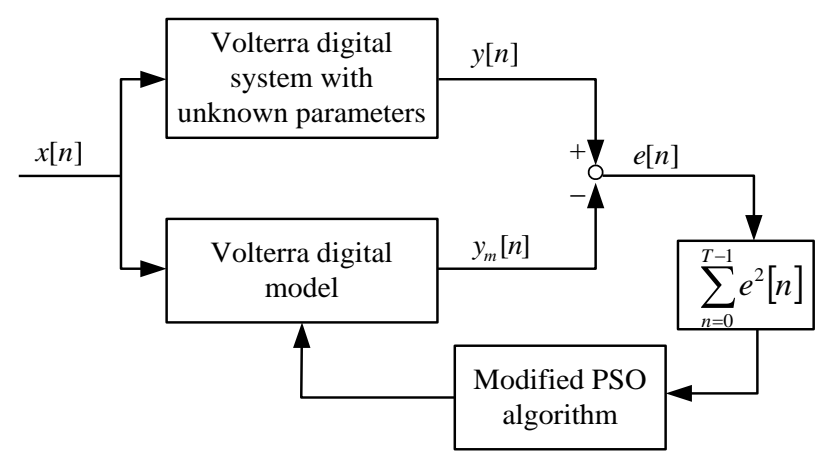

Fig. 1. Block diagram of the proposed estimation method.

Based on the modified PSO, the complete design steps for the parameter estimation of Volterra digital system can be outlined as follows.

Data: A series of input-output signal pairs $\{x[n], y[n]\}$, inertial weight $w$ and positive constants $c_{1}, c_{2}$, and $c_{3}$ in (7), sampling number $T$ in (8), population size $H$, number of subpopulations $L$, number of algorithm iterations $G$.

Goal: Estimate the unknown parameters of the Volterra digital system using the modified PSO algorithm.

1) Create an initial population consisting of $H$ particles from the interval $[-1,1]$ randomly and uniformly.

2) Partition the original population into $L$ subpopulations by 
the particle order.

3) If the assigned number of iterations $G$ is achieved, then the algorithm stops.

4) Calculate the objective function of each particle by (8) and record the individual best, the local best, and the global best.

5) Execute the modified velocity formula of (7) and the position formula of (6) for each particle.

6) Go back to Step III.

\section{Simulation Results}

In this section, an illustrative example for parameter estimations of Volterra digital system is demonstrated. The Volterra system considered is expressed by $y[n]=2+0.2 x[n]-1.2 x[n-1]+0.43 x[n-3]$

$$
+0.1 x[n] x[n-1]-0.7 x[n-1] x[n-2],
$$

where all the system parameters are assumed to be unknown. According to the structure of (9), a Volterra digital model which estimates the unknown system parameters can be given by

$$
\begin{aligned}
& y_{m}[n]=\theta_{1}+\theta_{2} x[n]+\theta_{3} x[n-1]+\theta_{4} x[n-3] \\
& +\theta_{5} x[n] x[n-1]+\theta_{6} x[n-1] x[n-2],
\end{aligned}
$$

where $\Theta=\left[\theta_{1}, \theta_{2}, \cdots, \theta_{6}\right]$ is the determined parameter vector and is also the particle of the algorithm. It is expected that the particle is adjusted by the proposed algorithm so that the error signal between $y[n]$ and $y_{m}[m]$ is minimized and the unknown parameters of (9) can be correctly solved.

TABLE I: THE RELATED VARIABLES USED IN THE PROPOSED ALGORITHM

\begin{tabular}{|c|c|c|c|c|}
\hline $\begin{array}{c}\text { Inertia weight } \\
w\end{array}$ & $\begin{array}{c}\text { Positive constants } \\
c_{1}, c_{2}, c_{3}\end{array}$ & $\begin{array}{c}\text { Sampling number } \\
T\end{array}$ & $\begin{array}{c}\text { Population size } \\
H\end{array}$ & $\begin{array}{c}\text { Number of subpopulations } \\
L\end{array}$ \\
\hline 0.8 & 1.0 & 50 & 50 & 5 \\
\hline
\end{tabular}

TABLE II: FINAL PARAMETER ESTIMATION RESULTS FOR RUN $1 \sim$ RUN 5

\begin{tabular}{|c||c|c|c|c|c|c|}
\hline & $\theta_{1}$ & $\theta_{2}$ & $\theta_{3}$ & $\theta_{4}$ & $\theta_{5}$ & $\theta_{6}$ \\
\hline \hline Run 1 & 2 & 0.2 & -1.2 & 0.43 & 0.1 & -0.7 \\
\hline Run 2 & 2 & 0.2 & -1.2 & 0.43 & 0.099999 & -0.700001 \\
\hline Run 3 & 2 & 0.2 & -1.2 & 0.43 & 0.1 & -0.699999 \\
\hline Run 4 & 2 & 0.2 & -1.2 & 0.43 & 0.1 & -0.700001 \\
\hline Run 5 & 2 & 0.2 & -1.2 & 0.43 & 0.1 & -0.7 \\
\hline
\end{tabular}

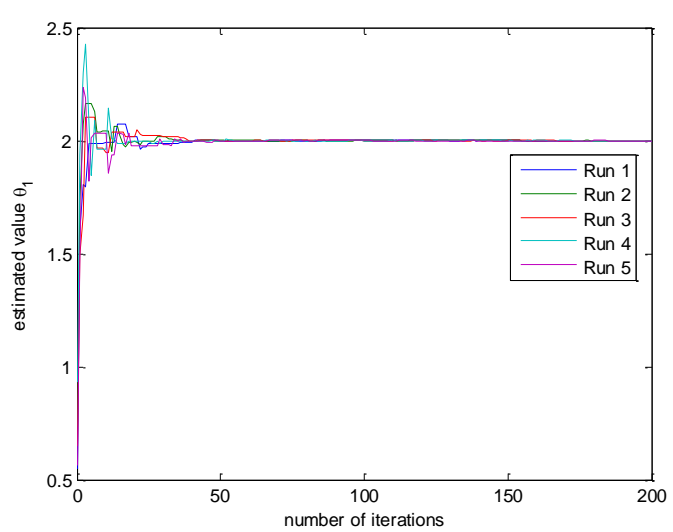

Fig. 2. Convergence curve of parameter $\theta_{1}$.

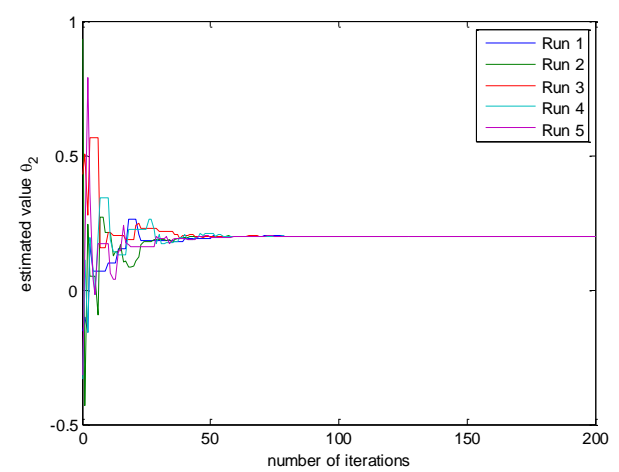

Fig. 3. Convergence curve of parameter $\theta_{2}$.

Table I lists the related variables used in the proposed algorithm for all the simulations. The external input signal $x[n]$ is generated from the interval $[-1,1]$ randomly to excite both the system of (9) and the model of (10). Moreover, five different sets of algorithm initial conditions, Run 1-Run 5, are examined to verify the algorithm robustness and feasibility. Simulation results are listed in Table II and plotted in Figs. 2-7, respectively. Table II lists the parameter estimation results of $\theta_{1}-\theta_{6}$ for Run 1-Run 5. It is found that all the system parameters can be accurately estimated. Their convergence curves with respect to the number of iterations are further shown in Figs. 2-7 for parameters $\theta_{1}-\theta_{6}$. As can be seen from these figures, they almost converges to the correct values after executing about 50 iterations.

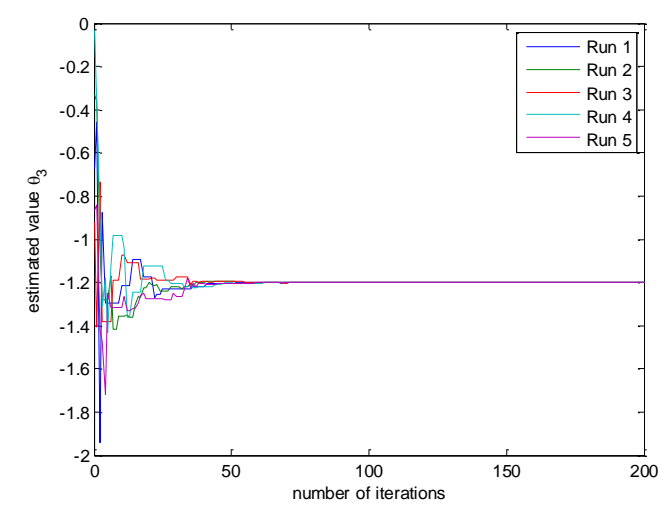

Fig. 4. Convergence curve of parameter $\theta_{3}$.

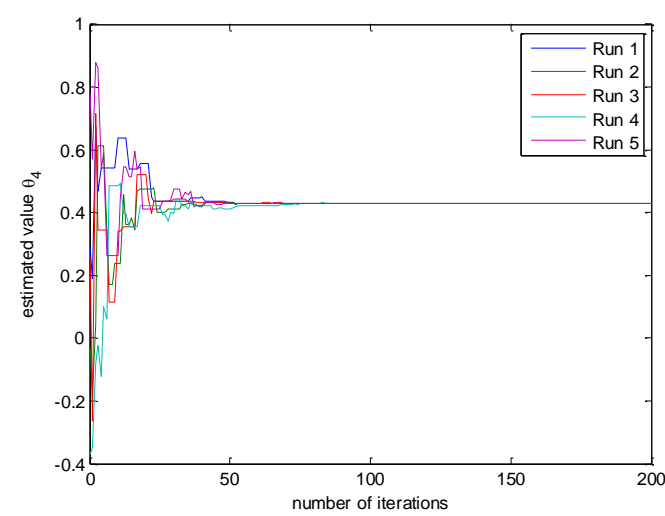

Fig. 5. Convergence curve of parameter $\theta_{4}$. 


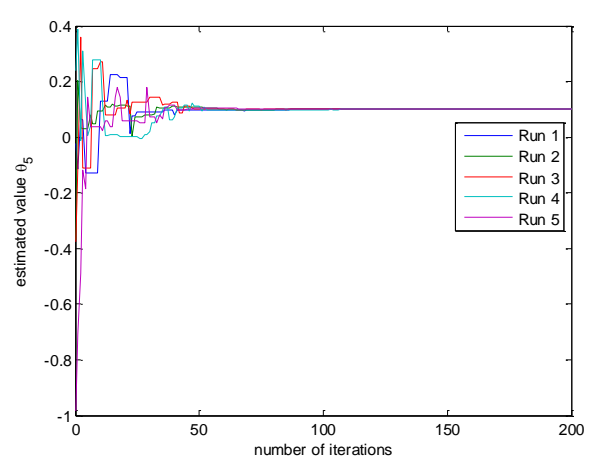

Fig. 6. Convergence curve of parameter $\theta_{5}$.

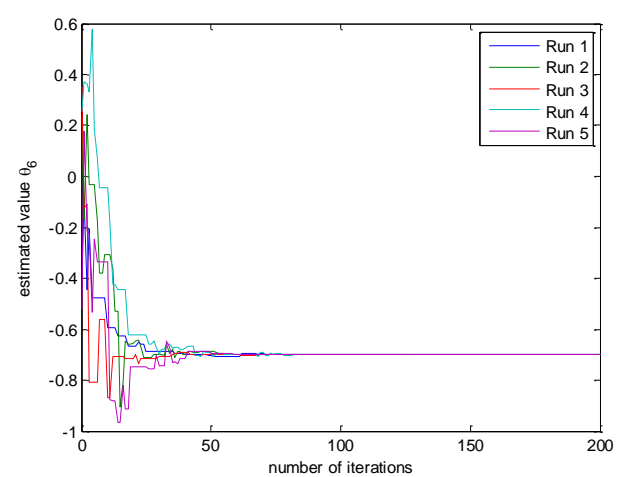

Fig. 7. Convergence curve of parameter $\theta_{6}$.

\section{CONCLUSIONS AND FUTURE WORK}

In this paper, we have successfully proposed a new parameter estimation algorithm which is based on the modified PSO for the Volterra digital system. A complete design strategy for parameter estimations of such digital system is clearly given. Following these design steps, the unknown system parameters can be correctly derived. The robustness and feasibility of the developed algorithm is also confirmed by testing different sets of initial conditions. For the future work, the proposed method can be utilized and extended to parameter estimations of another different kinds of digital systems or to the control system design.

\section{REFERENCES}

[1] Y. Mao, F. Ding, A. Alsaedi, and T. Hayat, "Adaptive filtering parameter estimation for Hammerstein nonlinear systems," Signal Processing, vol. 128, pp. 417-425, 2016.

[2] W. Cui, S. Wu, J. Tian, D. Liu, and S. Wu, "Parameter estimation for maneuvering targets with complex motion via scaled double-autocorrelation transform," Digital Signal Processing, vol. 59, pp. 31-48, 2016.

[3] Z. Hashemifard, H. Amindavar, and A. Amini, "Parameter estimation for continuous-time heavy-tailed signals modeled by $\alpha$-stable autoregressive processes," Digital Signal Processing, vol. 57, pp. 79-92, 2016.

[4] D. Wang and F. Ding, "Parameter estimation algorithms for multivariable Hammerstein CARMA systems," Information Science, vol. 355-356, pp. 237-248, 2016.
[5] J. S. Chang, C. C. Li, W. L. Liu, and J. H. Deng, "Two-stage parameter estimation applied to ordinary differential equation models," Journal of the Taiwan Institute of Chemical Engineers, vol. 57, pp. 26-35, 2015.

[6] Z. Xu, N. Qi, and Y. Chen, "Parameter estimation of a three-axis spacecraft simulator using recursive least-squares approach with tracking differentiator and extended Kalman filter," Acta Astronautica, Vol. 117, pp. 254-262, 2016.

[7] J. Kennedy and R. Eberhart, "Particle swarm optimization," in Proc. the IEEE International Conference on Neural Networks, vol. IV, Perth, Australia, 1995, pp. 1942-1948.

[8] D. H. Tungadio, J. A. Jordaan, and M. W. Siti, "Power system state estimation solution using modified models of PSO algorithm: Comparative study," Measurement, vol. 92, pp. 508-523, 2016.

[9] P. Porwik, R. Doroz, and T. Orczyk, "Signatures verification based on PNN classifier optimised by PSO algorithm," Pattern Recognition, vol. 60, pp. 998-1014, 2016.

[10] Z. Yuguang, A. Bo, and Z. Yong, "A PSO algorithm for multi-objective hull assembly line balancing using the stratified optimization strategy," Computer\& Industrial Engineering, vol. 98, pp. $53-62,2016$.

[11] H. Garg, "A hybrid PSO-GA algorithm for constrained optimization," Applied Mathematics and Computation, vol. 274, pp. 292-305, 2016.

[12] W. D. Chang and S. P. Shih, "PID controller design of nonlinear systems using an improved particle swarm optimization approach," Communications in Nonlinear Science and Numerical Simulation, vol. 15, pp. 3632-3639, 2010

[13] Y. S. Yang, W. D. Chang, and T. L. Liao, "Volterra system-based neural network modeling by particle swarm optimization approach," Neurocomputing, vol. 82, pp. 179-185, 2012.

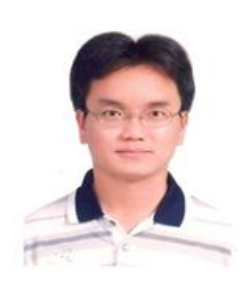

W. D. Chang received his Ph.D. degree in electrical engineering from National Sun Yat-Sen University, Kaohsiung, Taiwan in 2002. He is now a professor at the Department of Computer and Communication, Shu-Te University, Kaohsiung, Taiwan. His research interests include the intelligent signal processing, evolutionary computations, chaotic secure communication, and control engineering.

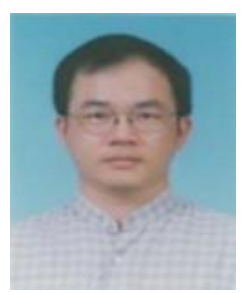

C. L. Chi received his Ph.D. degree in electrical engineering from National Cheng-Kung University, Tainan, Taiwan. He is now an associated professor at the Department of Computer and Communication, Shu-Te University, Kaohsiung, Taiwan. His research interests include the error control coding and wireless communication

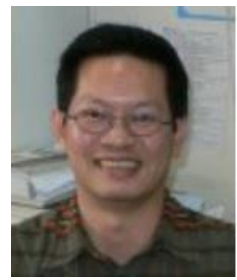

S. P. Shih received his Ph.D. degree in Graduate Institution of Space Science from National Central University, Taoyuan, Taiwan. He is now an assistant professor at the Department of Computer and Communication, Shu-Te University, Kaohsiung, Taiwan. His research interests include the communication system theory and satellite signal analysis.

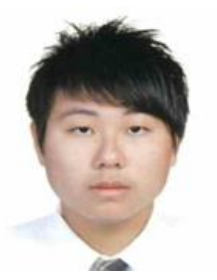

B. H. Ye is now a master student at the Department of Computer and Communication, Shu-Te University, Kaohsiung, Taiwan. His research interests are the digital filter design and evolutionary computations. 